\title{
Uso de gel de Aloe vera en la producción de plántulas de Capsicum chinense
}

\author{
Use of Aloe vera gel in Capsicum chinense seedlings production
}

\begin{abstract}
Tucuch-Haas CJ', Cen-Caamal JC', Kancab-Uc RA², Tucuch-Haas J/3*
Tecnológico Nacional de México/ITS del Sur del Estado de Yucatán, Carretera Muna-Felipe Carrillo Puerto Tramo OxcutzcabAkil, Km 41+400, Oxcutzcab, Yucatán, México.

2 Centro de Investigación en Alimentación y Desarrollo A.C. Carretera la Victoria Km. 06 Hermosillo, Sonora, México.

3 Instituto Nacional de Investigación Forestal Agrícola y Pecuario. Campo Experimental Mococha, Km 25 Carretera MéridaMotul, Yucatán, México.
\end{abstract}

\section{RESUMEN}

La producción de chile habanero en Yucatán, demanda plántulas de calidad al momento del trasplante, lo que ha llevado al uso de reguladores de crecimiento, que si bien es cierto potencializan la germinación y desarrollo de las plantas, también incrementan los costos de producción. Por tal motivo se están buscando alternativas de producción que reduzcan los costos, razón por la cual, se ha puesto la mirada en el gel de la sábila, cuya composición química reporta la presencia de fitorreguladores como las giberelinas y el ácido salicílico. Para estudiar la respuesta del gel de sábila en la emergencia y desarrollo de la raíz y vástago en plántulas de chile habanero, se sembraron 500 semillas de chile habanero variedad "Mayapan", en grupos de 100, previamente embebidas en $100 \mathrm{~mL}$ de gel de sábila al 0,2.5, 5, 10 y $20 \%$, durante $24 \mathrm{~h}$. Los resultados obtenidos demuestran que el gel de sábila reduce cuatro días la emergencia y favorece hasta en un $18 \%$ el desarrollo radicular, la altura de la planta y biomasa fresca total; $11 \%$ el diámetro del tallo y $13 \%$ número de hojas, observándose un mayor efecto con la solución al 5 \% de gel de sábila.

Palabras clave: Fitorregulador, sábila, chile habanero, imbibición.

\section{ABSTRACT}

The production of habanero pepper in Yucatán demands quality seedlings at the time of transplantation, which has led to the use of growth regulators, which, although it is true, potentiate the germination and development of the plants, they also increase production costs. For this reason, production alternatives are being sought that reduce costs, which is why an eye has been focused on Aloe vera gel, whose chemical composition reports the presence of phytoregulators such as gibberellins and salicylic acid. To study the response induced by the aloe gel in the emergence and development of the root and shoot in habanero pepper seedlings, 500 seeds of the habanero pepper variety "Mayapan" were sown, in groups of 100 , previously soaked in $100 \mathrm{~mL}$ of aloe gel at $0,2.5,5,10$ and $20 \%$, for $24 \mathrm{~h}$. The results obtained show that Aloe vera gel reduces emergence by four days and favors root development, plant height and total fresh biomass by up to $18 \%$, also $11 \%$ the diameter of the stem and $13 \%$ number of leaves, observing a greater effect with the $5 \%$ solution of Aloe vera gel.

Keywords: Phytoregulator, Aloe vera, habanero pepper, imbibition.

\section{INTRODUCCIÓN}

A nivel mundial México ocupa el segundo lugar en la producción de frutos de género Capsicum, con un volumen promedio de los últimos diez años de 2,785,000 toneladas anuales (SIAP, 2020). En particular para la especie $C$. chinense (chile habanero) cuya principal región de producción es el sur del país, en la que destaca el estado de Yucatán como el primer lugar de producción, se reporta un promedio de producción de 9, 529.8 toneladas (SIACOM, 2020) que va en aumento.

C. chinense posee gran diversidad de usos en la industria química, alimentaria y farmacéutica gracias a sus bondades nutricionales y terapéuticas dado su nivel de pungencia (Flores-López y Sánchez-Osorio, 2020), aunado a su denominación de origen otorgado en el 2010 por el Instituto Mexicano de la Propiedad Industrial IMPI (DOF, 2010), han sido los detonantes claves en los incrementos en la demanda de esta hortaliza, lo que ha llevado a expertos en el área a la búsqueda de alternativas, que garanticen el buen desarrollo de las plantas e incrementen el rendimiento del fruto.

Una práctica común en la actualidad, dentro del sistema producción de chile habanero, es el empleo de almácigos en charolas de poliestireno (Gonzáles et al., 2018) con la cual se busca un alto porcentaje de germinación de las semillas y buen vigor de las plántulas al momento del trasplante (Castillo-Aguilar et al., 2015), mediante el control de condiciones de luz, humedad, aporte de nutrientes y problemas fitosanitarios (Preciado et al., 2002; Mendoza-Paredes et al., 2021). Entre otras prácticas, además del uso de bacterias promotoras de crecimiento vegetal (Adame-García et al., 2021), que se emplea de manera adicional, como herramienta para garantizar la germinación, mayor vigor y capacidad de resistir el estrés del trasplante, se encuentra el uso de fitorreguladores o reguladores de crecimiento vegetal. Al respecto Castro et al. (2019) mencionan que la adecuada selección y aplicación de los fitorreguladores durante el cultivo de plantas de interés agrícola, representa una importante herramienta

*Autor para correspondencia: Jorge Ismael Tucuch Haas Correo electrónico: leamsi182@hotmail.com

Recibido: 28 de julio de 2021

Aceptado: 7 de enero de 2022 
para mejorar algunos de los aspectos de mayor interés en su producción.

Si bien existen diversos productos a base de fitorreguladores sintéticos en el mercado, disponibles para su uso (Ramírez-Luna et al., 2005), estos son pocos disponibles en las comunidades más apartadas de las ciudades, además eleva los costos de producción e impacta directamente al bolsillo del productor. Sin embargo, algunos órganos de especies vegetales, dado su alta concentración de fitorreguladores presentan un potencial uso como estimulante en el desarrollo de otras especies vegetales (Carranza et al., 2021), tal es el caso de Aloe vera. De acuerdo con Domínguez-Fernández (2012) el gel de Aloe vera dentro de su composición química posee fitorreguladores, tales como las giberelinas y ácido salicílico, esta primera responsable de la ruptura de la dormancia o latencia de las semillas principalmente (Li et al., 2015) y este último de la protección contra patógenos (Lu et al., 2016), entre otras funciones como el favorecimiento en el desarrollo de plántulas (Tucuch et al., 2016).

Evidencias de la acción del gel de Aloe vera como regulador de crecimiento vegetal han sido reportado por Boschi et al. (2017) al evaluar su aplicación a estaquillas de Origanum vulgare para propagación, donde observaron mayor número de raicillas y longitud de estas. Por otro lado, su uso en medios de cultivos para la propagación in vitro ha demostrado mejor respuesta en la formación de raíces, incluso superó a los reguladores de crecimiento tradicionales tales como AIA y AIB (Pulido y Becerra, 2016).

Dado las necesidades de producción de plántulas de calidad y las evidencias planteadas del gel de Aloe vera, respecto a su función como regulador de crecimiento vegetal y puesto que no existe reportes de su aplicación en el cultivo de chile habanero, el presente trabajo tuvo como objetivo evaluar la respuesta de este compuesto en la germinación y desarrollo de plántulas de chile habanero en semilleros.

\section{MATERIALES Y MÉTODOS}

El experimento se llevó a cabo en las instalaciones del Instituto Tecnológico Superior del Sur del Estado de Yucatán (ITSSY), en un invernadero tipo túnel de $500 \mathrm{~m}^{2}$. Como material vegetal se utilizaron semillas de chile habanero variedad Mayapan.

Las pencas de sábila, para la extracción del gel se colectaron en el "Instituto Tecnológico Superior del Sur del Estado de Yucatán", específicamente en áreas prácticas de dicho instituto, para la cual se tomaron en cuenta aspectos como la altura, el diámetro (grosor de la penca) y sobre todo el estado de madurez. Se cortaron las hojas inferiores, cercanas a la base de la planta. Una vez obtenidas las pencas de sábila se procedió a su desinfección, al igual que los materiales (probeta de $1 \mathrm{~L}$ y $100 \mathrm{~mL}$, vaso de precipitado $1 \mathrm{~L}$, agitador, cuchillo), con una solución de cloro al $2 \%$.

Para la preparación de los tratamientos $(2.5,5.0,10.0$ y $20.0 \%$ de gel se sábila), se procedió a extraer el gel de las pencas (Figura 1), mediante el método del fileteado con la finalidad de extraer en su totalidad el gel. Para la obtención

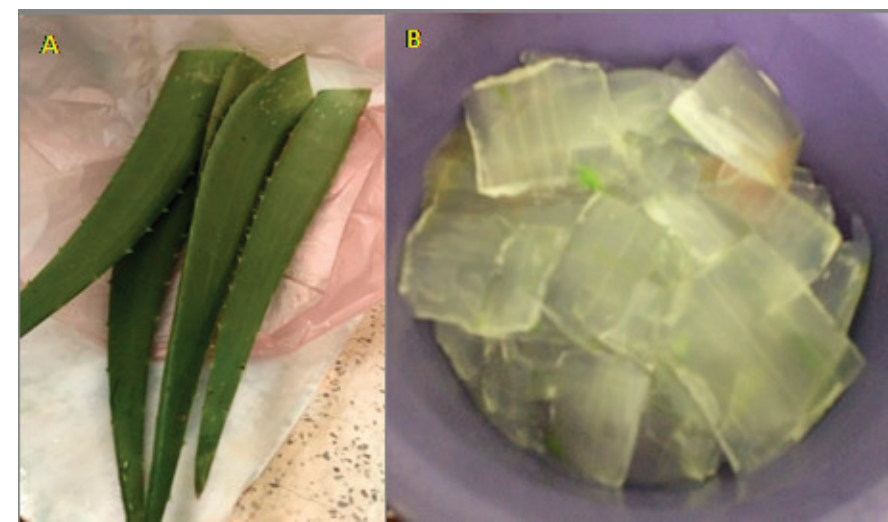

Figura 1. Pencas de Aloe vera seleccionada (A) y gel extraído (B) para la elaboración de los tratamientos.

Figure 1. Aloe vera stalks selected for the extraction of the gel $(A)$ and the extracted gel (B) for the elaboration of the treatments.

de los tratamientos se pesaron 25, 50, 100 y $200 \mathrm{~g}$ de gel de sábila, con la ayuda de una balanza analítica, mismas que se licuaron en $500 \mathrm{~mL}$ de agua y retiraron las impurezas con un tamiz de $1 \mathrm{~mm}$ de diámetro, finalmente se aforaron a $1 \mathrm{~L}$ con agua y colocaron en envases de plásticos con capacidad de $1 \mathrm{~L}$.

Previo al suministro de los tratamientos a las semillas se les realizó una desinfección, para tal efecto se sumergieron las semillas en $100 \mathrm{~mL}$ de una solución de cloro al 1\%, durante cinco minutos, para posteriormente hacerle dos enjuagues con $50 \mathrm{~mL}$ de agua destilada.

Terminada la desinfección y separadas en grupos de 100 semillas, se embebieron en $100 \mathrm{~mL}$ de sus respectivos tratamientos $[2.5,5.0,10,20 \%$ de GS y un control (0.0\%)] durante $24 \mathrm{~h}$. Transcurrido este tiempo, se llevó acabo la siembra, en charolas de unicel de 200 cavidades, previamente desinfectadas con una solución de hipoclorito al $2 \%$. Como sustrato se utilizó $10 \mathrm{Kg}$ de Peat Moss y $4 \mathrm{~L}$ de agua para humedecer.

Posterior a la germinación, se aplicaron riegos de a 5 $\mathrm{mL}$ por cavidad cada tercer día, con la ayuda de una jeringa, para obtener un riego uniforme y se rotaron las charolas todos los días para asegurar que todas las plántulas recibieran la misma cantidad de luz solar, cabe mencionar que las rotaciones se realizaron dos veces al día. Una vez acomodadas las charolas dentro del invernadero y que las plántulas presentaron las primeras hojas verdaderas bien definidas, se inició con la fertilización (10 días después de la emergencia) para posteriormente continuar cada tercer día, con la ayuda de una jeringa ( $5 \mathrm{~mL}$ planta-1 ${ }^{-1}$, con un suministro de $0.22 \mathrm{~g}$ $\mathrm{L}^{-1}$ de urea, $0.33 \mathrm{~g} \mathrm{~L}^{-1}$ de MAP y $0.40 \mathrm{~g} \mathrm{~L}^{-1}$ de NKS y $1 \mathrm{~mL}$ de poliquel multi en $10 \mathrm{~L}$ de agua.

Independiente a la imbibición de las semillas a los 14 y 21 días después de la siembra se aplicaron los mismos tratamientos, de manera foliar, con la ayuda de un atomizador, hasta punto de goteo (300 mL por tratamiento).

La emergencia de las plántulas se evaluó a partir de los 4 días y hasta los 15 días después de la siembra, para ello, se cuantificó diariamente el número de cavidades con cotiledones expuestas en la superficie del sustrato. La altura 
de la planta, el diámetro del tallo y la longitud de la raíz se evaluaron a los 31 días después de la siembra, para la altura se utilizó una regla milimétrica; los puntos de referencia para hacer la medición fueron la base del tallo y la yema apical de la planta; el diámetro del tallo se realizó a $5 \mathrm{~cm}$ de la base proximal al sustrato mediante un vernier digital; mientras que la longitud de la raíz se determinó de la base del tallo hasta el ápice de la raíz principal.

Entre otras variables se evaluó el peso fresco y seco de la parte aérea y de la raíz: Para obtener el peso fresco, se sacaron las plántulas tomadas al azar de cada tratamiento de las charolas (15 plantas por tratamiento) y mismas a las que se le lavaron la raíz, hasta dejarlo libre de sustrato; posteriormente se dividió la planta en hojas, tallo y raíz, y por último con una balanza analítica se cuantificó los pesos de cada órganos y biomasa fresca total. Una vez tomado los datos del peso fresco, las muestras se metieron en bolsas de papel para poder secarlas en una estufa a una temperatura de $60^{\circ} \mathrm{C}$, cuando alcanzaron un peso constante ( $72 \mathrm{~h}$ ), con la balanza analítica se cuantificó el peso seco de la hoja, tallo y raíz de todas las muestras.

Se empleó un diseño completamente al azar con cuatro tratamientos más un control, con 15 repeticiones y una planta como unidad experimental. Los resultados fueron analizados mediante el análisis de varianza y cuando se detectaron diferencias estadísticas se realizó la comparación de medias por el método Tukey $(P \leq 0.05)$ con el paquete estadístico SAS (2004).

\section{RESULTADOS Y DISCUSIÓN Emergencia}

La respuesta presentada por acción del GS en la emergencia de plántulas de chile habanero se reporta en la Figura 2 , el cual permite señalar que las semillas de chile habanero respondieron de manera significativa a los tratamientos de GS. El inicio de la emergencia de todos los tratamientos, con excepción de la concentración 10 \% de GS, que ocurrió un día después, se manifestaron a los $6 \mathrm{dds}$, mientras que la del control ocurrió a los 11 dds. El vigor de las semillas considerado como el tiempo necesario para que emerjan el $50 \%$ de las plántulas, se vio favorecida con todos los tratamientos, dado que este valor se alcanzó cuatro días previos al del control; de igual forma la viabilidad, determinada como el porcentaje de semillas que son capaces de germinar, aumentó con los tratamientos, observándose un mayor efecto con la concentración $5 \%$ de GS, que obtuvo el $97 \%$ de germinación frente al control que fue de $80 \%$.

La respuesta favorable del GS a la emergencia, puede ser atribuido a la presencia de giberelinas en su composición química (Domínguez-Fernández et al., 2012), los cuales son responsables de dar inició a la germinación (Alcantara et al., 2019), al respecto Pérez y Pita (1998) mencionan que las giberelinas son capaces de promover la germinación de semillas tanto durmientes como no durmientes. Entre otros compuestos se encuentran algunas enzimas importantes como las lipasas y amilasas, responsables de la degradación

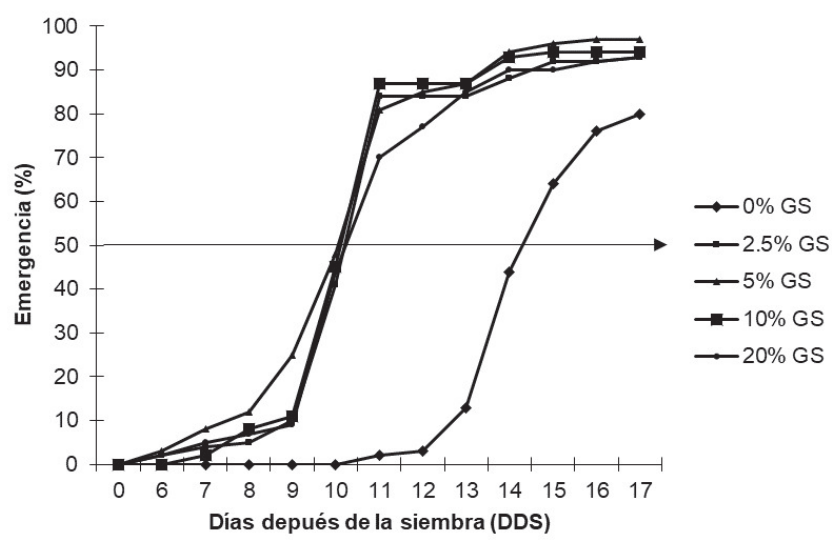

Figura 2. Patrón de respuesta en la dinámica de emergencia por efecto de diferentes porcentajes del gel de sábila (GS).

Figure 2. Response pattern in the emergence dynamics of habanero pepper seedlings due to the effect of different concentrations of Aloe vera gel (GS).

de lípidos y almidón respectivamente, almacenadas como reserva, para la obtención de la energía necesaria para la activación del metabolismo de la semilla; además de la glucosa y aminoácidos, que también participan en el suministro de energía necesaria para el metabolismo de las semillas (Pérez y Pita, 1998; Domínguez-Fernández et al., 2012).

\section{Altura de planta, diámetro del tallo y longitud de la raíz}

Tanto la altura del vástago, como el diámetro del tallo y la longitud de la raíz incrementaron significativamente en un $18.3,12.5$ y $14.2 \%$ respectivamente comparado con el control, por el efecto del GS al 5 \% (Figura 3), mientras que, para el resto de los tratamientos, aunque superaron al control no fueron estadísticamente significativos. Este comportamiento sugiere que $50 \mathrm{~g}$ del gel diluidas en un litro de agua, son suficientes para inducir un efecto significativo en el desarrollo de las plantas y da la pauta para sugerir, que el efecto presentado, es por acción de los fitorreguladores presentes, como se sabe, por lo general estos ejercen su función a muy bajas concentraciones y cuyo principal efecto se produce a nivel celular, donde cambian los patrones de crecimiento de los vegetales y permiten su control (Alcantara et al., 2019).

Respecto al diámetro del tallo (Figura 3) se puede apreciar que al igual que para la altura, el tratamiento $5 \%$ de GS fue el que mejor resultado presentó, el cual superó al control y al tratamiento $20 \%$ de GS, no así para $2.5 \%$ y 10 $\%$ de GS, los cuales fueron estadísticamente iguales, aunque sus valores se encontraron ligeramente por debajo de esta.

Por otro lado (Figura 3), se pudo determinar que diferente al comportamiento de los valores de altura de planta y diámetro del tallo, en la cual el suministro de $5 \%$ de GS presentaron los mejores resultados, en la longitud de la raíz, la mayor sensibilidad se presentó cuando se utilizó GS con una dosis de $2.5 \%$, sin embargo, no fueron estadísticamente diferentes a la concentración del $5 \%$, pero si con el resto de los tratamientos y el control. Este comportamiento sugiere que la raíz presenta una sensibilidad más marcada a bajas contracciones, por lo que los niveles y sensibilidad de los 

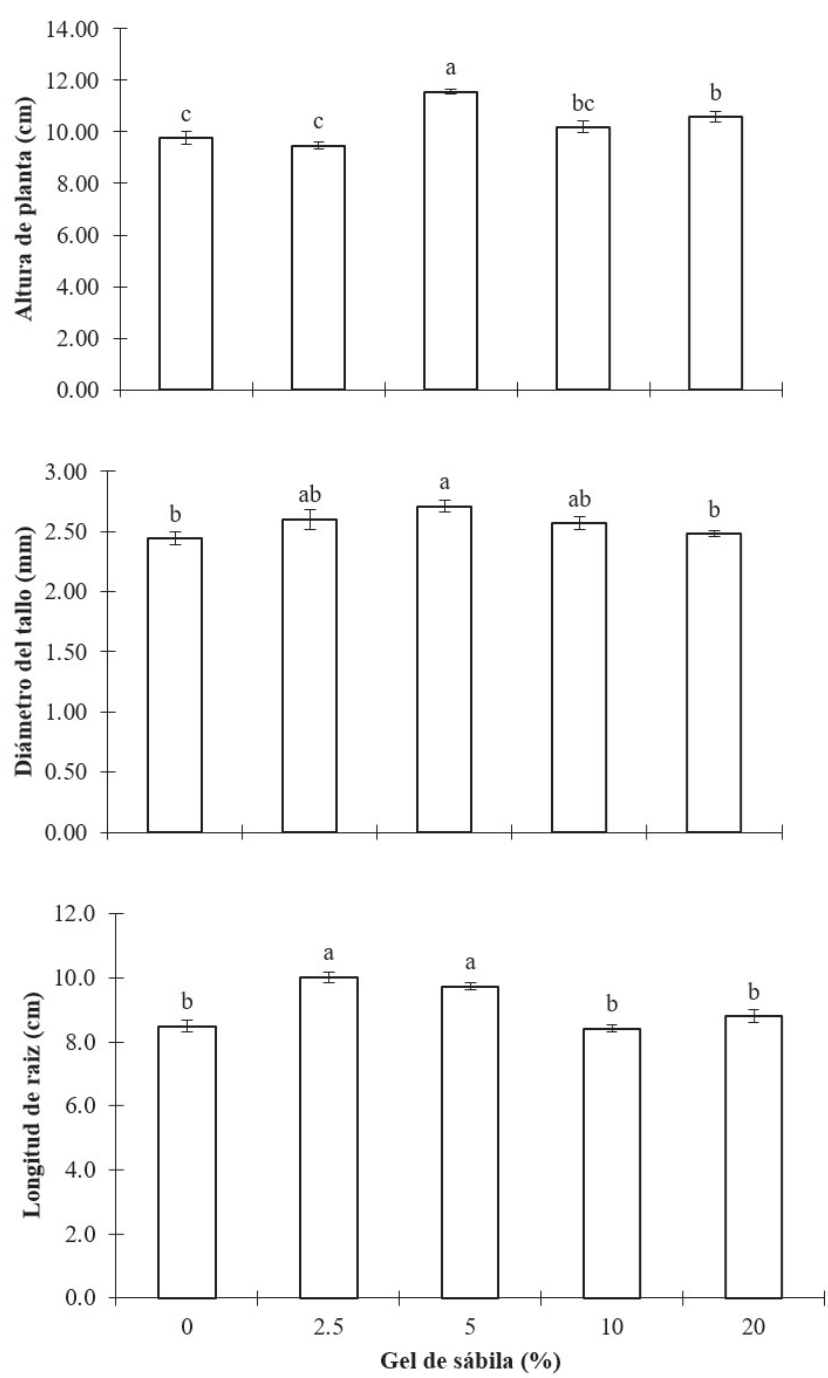

Figura 3. Patrones de respuesta en la altura de la planta, diámetro del tallo y longitud de la raíz al efecto de diferentes concentraciones de gel de sábila en plántulas de chile habanero (Capsicum chinense), cada punto es la media de 15 repeticiones \pm error estándar. Valores con la misma letra dentro de columnas son iguales de acuerdo con la prueba de Tukey a una $\mathrm{P} \leq 0.05$.

Figure 3. Response patterns in plant height, stem diameter and root length, to the effect of different concentrations of Aloe vera gel in habanero pepper (Capsicum chinense) seedlings, each point is the mean of 15 repetitions \pm error standard. Values with the same letter within columns are equal according to Tukey's test at $\mathrm{P} \leq 0.05$.

tejidos juegan un papel importante en la expresión de la respuesta.

\section{Biomasa fresca y seca}

En la Tabla 1 se reporta el efecto de GS en el número de hojas, biomasa seca y fresca total. El número de hojas presentó diferencias significativas para todos los tratamientos respecto al control, no así entre tratamientos; la máxima diferencia encontrada fue $12 \%$ con el tratamiento de $5 \%$ de GS. Respecto a la biomasa fresca total solo el tratamiento con $2.5 \%$ de GS presentó diferencia significativa, incrementándose en un $18 \%$ por encima del control; mientras que para la biomasa seca total los tratamientos 5 y $10 \%$ de GS,
Tabla 1. Efecto de diferentes porcentajes de gel de sábila en la biomasa de plántulas de chile habanero.

Table 1. Effect of different percentages of Aloe vera gel on the biomass of habanero pepper seedlings.

\begin{tabular}{cccc}
\hline Trat (\% GS) & NH (\#) & BFT (g) & BST (g) \\
\hline 0 & $6.6 \pm 0.16 \mathrm{~b}$ & $1.22 \pm 0.025 \mathrm{~b}$ & $0.126 \pm 0.002 \mathrm{~b}$ \\
2.5 & $7.4 \pm 0.17 \mathrm{a}$ & $1.45 \pm 0.073 \mathrm{a}$ & $0.101 \pm 0.008 \mathrm{ab}$ \\
5 & $7.5 \pm 0.16 \mathrm{a}$ & $1.40 \pm 0.042 \mathrm{ab}$ & $0.159 \pm 0.004 \mathrm{a}$ \\
10 & $7.4 \pm 0.16 \mathrm{a}$ & $1.37 \pm 0.047 \mathrm{ab}$ & $0.138 \pm 0.007 \mathrm{a}$ \\
20 & $7.4 \pm 0.16 \mathrm{a}$ & $1.37 \pm 0.031 \mathrm{ab}$ & $0.126 \pm 0.005 \mathrm{ab}$ \\
\hline
\end{tabular}

$\mathrm{GS}=$ gel de sábila; $\mathrm{NH}=$ número de hoja; $\mathrm{BFT}=$ biomasa fresca total;

BST=biomasa seca total. Cada valor es la media de 15 repeticiones. Valores con la misma letra dentro de columnas son iguales de acuerdo con la prueba de Tukey a una $\mathrm{P} \leq 0.05$

superaron significativamente al control en un 26 y $15 \%$ respectivamente.

Las repuesta a la sensibilidad de Aloe vera, principalmente en la raíz y la altura, es coincidente con lo reportado por Boschi et al. (2017) quienes documentaron un mayor número de raicillas y longitud del tallo en estaquillas de Origanum vulgare para propagación, con un aporte de 150 $\mathrm{g}$ de gel por litro de agua; $\mathrm{y}$ los resultados en otras entras especies vegetales con la adición de una proporción de este compuesto en el medio de cultivo para propagación in vitro, donde se observó una repuesta significativa en el formación de raíces (Pulido y Becerra, 2016).

Se puede considerar que en el presente estudio la respuesta en el desarrollo que se aprecia por el efecto del GS (Figura 4), es el resultado de la interacción de los compuestos (aminoácidos, lípidos, enzimas y carbohidratos) y elementos químicos (Calcio, magnesio, potasio, zinc, sodio, cobre, hierro, manganeso, fósforo, cromo), esenciales presentes, que favorecen tal expresión a nivel de organismo (DomínguezFernández et al., 2012). Sin embargo, es preciso destacar que parte del efecto en el desarrollo de la planta puede ser atribuido al ácido salicílico presente, ya que este compuesto en particular es capaz de incrementar el nivel de división celular del meristemo apical, el tamaño de la cofia y las raíces laterales (Echeverría-Machado et al., 2007; Shakirova et al., 2003), ejerciendo cambios positivos en la morfología, longitud, área, volumen, perímetro y peso fresco y seco de la raíz, tal como se ha reportado en plantas de Glycine max L. (Gutiérrez-Coronado et al., 1998), Pinus patula (San-Miguel et al., 2003), Crysanthemum morifolium (Villanueva-Cohuo et al., 2009), Catharanthus roseus (Echeverría-Machado et al., 2007), Lycopersicon esculentum (Larqué-Saavedra et al., 2010) y Triticum aestivum L.

Trabajos como las de Rodríguez-Larramendi et al. (2017) y Dzib-Ek et al. (2021) también complementan la hipótesis de que el ácido salicílico presente en el gel de sábila, podría potencializar la germinación y el desarrollo de las plántulas, puesto que en su estudio de inhibición de semillas de frijol y tomate en diferentes dosis de ácido salicílico, encontraron beneficios significativos en estas variables. 


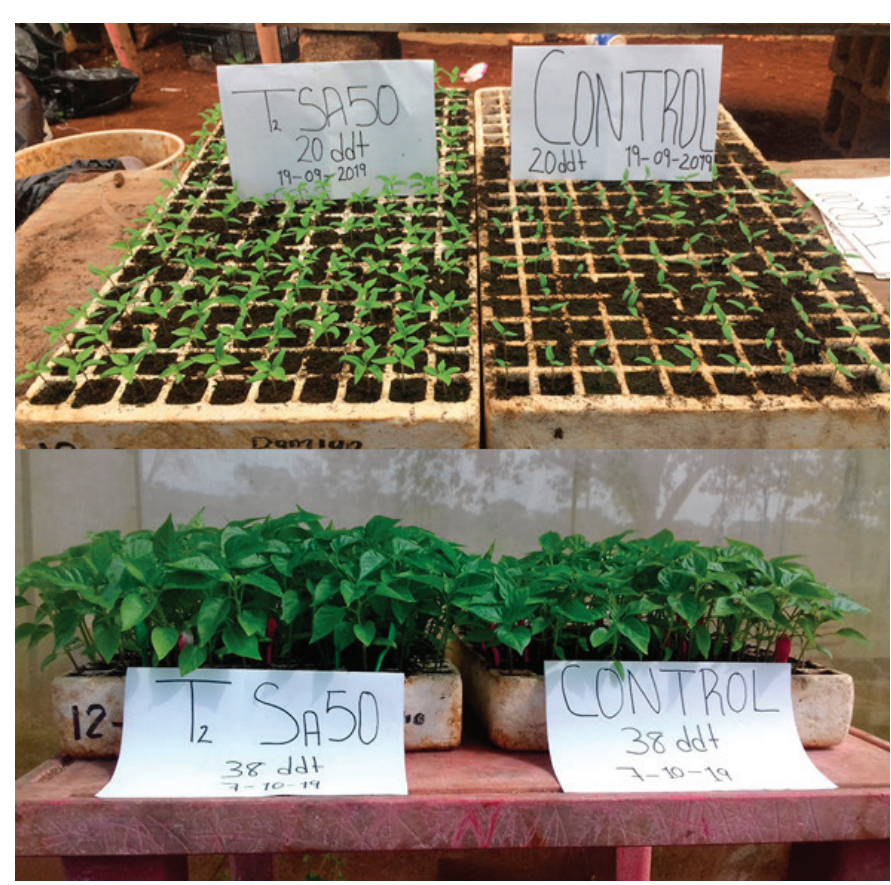

Figura 4. Desarrollo de las plántulas a los 20 y 38 días después de la siembra tratados con $5 \%$ de gel de sábila.

Figure 4. Seedling development at 20 and 38 days after sowing treated with $5 \%$ Aloe vera gel.

\section{CONCLUSIÓN}

La imbibición de las semillas de chile habanero en una solución con $5 \%$ de gel de sábila redujo cuatro días el inicio de la emergencia de las plántulas y la aplicación foliar favoreció hasta en un $18 \%$ el desarrollo radicular, la altura de la planta y biomasa fresca total; y 11 y $13 \%$ el diámetro del tallo y número de hojas, respectivamente.

\section{BIBLIOGRAFÍA}

Adame-García, J., Murillo-Cuevas, F.D., Flores-de la Rosa, F.R., Velázquez-Mendoza, V., López-Vázquez, M., Cabrera-Mireles, H. y Antonio-Vázquez, E. 2021. Identificación molecular y evaluación de bacterias en el desarrollo vegetativo y producción de chile habanero. Biotecnia. 23(3): 151-157.

Alcántara, C.J.S., Acero, G.J., Alcántara, C.J.D. y Sánchez M.R.M. 2019. Principales reguladores hormonales y sus interacciones en el crecimiento vegetal. NOVA. 17: 109-129.

Boschi, C.L., Gandolfo, E. y Vence, L. 2017. Evaluación de gel de Aloe vera en el enraizamiento de estaquillas de orégano (Origanum vulgare). Horticultura Argentina. 36(89): 06-16.

Castillo-Aguilar, C. de la C. 2018. Producción de planta de chile habanero (Capsicum chinense Jacq.). Agro Productividad. 8(4): 73-78.

Castro, R.J.J., Solís, O.M.M., Castro, R.R. y Calderón, V.C.L. 2019. Minireview: Uso de fitorreguladores en el manejo de cultivos agrícolas. Frontera biotecnológica. 13(3): 14-18.

Carranza-Álvarez, C., Trinidad-García, K.L., Reyes-Hernández, H., Castillo-Pérez L.J. y Fortanelli-Martínez, J. 2021. Efecto de extractos orgánicos naturales sobre la micropropagación de Vanilla planifolia Jacks. ex Andrews (Orchidaceae). Biotecnia. 23(1): 5-12.

DOF. 2010. Declaratoria general de protección de la denominación de origen del chile habanero de la península de Yucatán. [Consultado 30 julo 2020]. Disponible en: http://dof.gob.mx/nota_detalle.php?codigo=5145315\&fec ha $=04 / 06 / 2010$

Domínguez-Fernández, R.N., Arzate-Vázquez, I., Chanona-Pérez, J.J., Welti-Chanes, J.S., Alvarado-González, J.S.A., CalderónDomínguez, G., Garibay-Febles, V. y Gutiérrez-López, G.F. 2011. El gel de Aloe vera: Estructura, Composición química, procesamiento, actividad biológica e importancia en la industria farmacéutica y alimentaria. Revista Mexicana de ingeniería química. 11(1): 23-43.

Dzib-Ek, G., Villanueva-Couoh, E., Garruña-Hernández, R., Vergara, Y.S. y Larqué-Saavedra, A. 2021. Efecto del ácido salicílico en la germinación y crecimiento radicular del tomate. Revista Mexicana de Ciencias Agrícolas, 12(4): 73540.

Echevarría-Machado, I., Escobedo G.M. and Larqué-Saavedra A. 2007. Responses of transformed Catharanthus roseus roots to femtomolar concentrations of salicylic acid. Plant physiol. Biochem. 45: 501-507.

Flores, L.M.L. y Sánchez, O.E. 2020. Entorno productivo del chile habanero en la Península de Yucatán, México. En Metabolómica y cultivo del chile habanero (Capsicum chinense Jacq) de la Península de Yucatán (332). Mérida, Yucatán: CIATEJ.

González, T.E., Zúñiga, A.J. y Vázquez, F.F. 2018. Mejoramiento genético del chile habanero de la Península de Yucatán. CONACYT. Mérida, Yucatán, México. pp17-21.

Gutiérrez-Coronado, M.A., Trejo-López, C. and Larqué-Saavedra, A. 1998. Effects of salicylic acid on the growth of roots and shoots in soybean. Plant Physiol. Biochem. 36: 563-565.

Larqué-Saavedra A., Martín-Mex R., Nexticapan-Garcéz, A., Vergara-Yoisura S. y Gutiérrez-Rendón, M. 2010. Efecto del ácido salicílico en el crecimiento de plántulas de tomate (Lycopersicon esculentum Mill.). Rev. Chapingo Ser. Hortic. 16: 183-187.

Li, G., Liu, S., Sun, Z., Xia, L., Chen, G. y You, J. 2015. A simple and sensitive HPLC method based on pre-column fluorescence labelling for multiple classes of plant growth regulator determination in food samples. Food Chemistry. 170: 123130.

Lu, H., Greenberg, J.T. and Holuigue, L. 2016. Salicylic Acid Signaling Networks. Front. Plant Sci 7: 238. doi: 10.3389/ fpls.2016.00238.

Mendoza-Paredes, J.E., Castillo-González, A.M., Avitia-García, E., Valdez-Aguilar, L.A. y García-Mateos, M.R. 2021. Efecto de diferentes proporciones de luz, led azul: roja en plantas de chile habanero (Capsicum chinense Jacq.). Biotecnia. 23(1): 110-119.

Preciado, R.P., Baca, G.A, Tirado, J.L., Kohashi-Shibata, J., Tijerina, L., Martínez, A. 2002. Nitrógeno y potasio en la producción de plántulas de melón. Terra. 20:267-276.

Pita, V.J.M y Pérez, G.F. 1998. Germinación de semillas. Ministerio de Agricultura Pesca y Alimentación. Hojas divulgadoras/ Número 2090 HD. Madrid España.

Pulido, S.N. y Becerra, A.J.L. 2016. Aloe vera (Aloe barbadensis Miller) en la regeneración de explantes de Agra (Vaccinium meridionale Swartz). Cultura científica. 14: 58-68.

Ramírez-Luna, E., Castillo-Aguilar C. C., Aceves-Navarro E. y Carrillo-Ávila, E. 2005. Efecto de productos con reguladores de crecimiento sobre la floración y amarre de fruto en chile 'habanero'. Revista Chapingo Serie Horticultura. 11(1): 93-98. 
Rodríguez-Larramendi, L.A., González-Ramírez, M., GómezRincón, M.A., Guevara-Hernández, F., Salas-Marina, M. A. y Gordillo-Curiel, A. 2017. Efectos del ácido salicílico en la germinación y crecimiento inicial de plántulas de frijol (Phaseolus vulgaris L.). Revista De La Facultad De Agronomía De La Universidad Del Zulia. 34(3): 253-269.

San Miguel, R., Gutiérrez M., Larqué-Saavedra, A. 2003. Salicylic acid increases the biomass accumulation of Pinus patula. Southern Journal of Applied Forestry. 27: 52-54.SAS, 2004. Statistical Analysis System Institute. SAS Proceeding Guide, Version 8.1. SAS Institute. Cary, NC. USA

Shakirova, F.M., Sakhabutdinova A.R., Bezrukova M.V., Fatkhutdinova R.A. and Fatkhutdinova, D.R. 2003. Changes in the hormonal status of wheat seedlings induced by salicylic acid and salinity. Plant Sci. 164: 317-322.
SIACOM-NG. 2020. Módulo agrícola estatal. [Consultado 30 julio 2020]. Disponible en https://www.gob.mx/siap/ documentos/siacon-ng-161430

SIAP. 2020. Panorama agroalimentario 2020. [Consultado 30 julio 2020] Disponible en: https://nube.siap.gob.mx/gobmx publicaciones_siap/pag/2020/Atlas-Agroalimentario-2020

Tucuch-Haas, C.J., Alcántar-González, G., Volke-Haller, V.H., Salinas-Moreno, S., Trejo-Téllez, L.I. y Larqué-Saavedra, A. 2016. Efecto del ácido salicílico sobre el crecimiento de raíz de plántulas de maíz. Revista Mexicana de ciencias agrícolas. 7: 709-716.

Villanueva-Couoh, E., Alcántar-González, G., Sánchez-García, P., Soria-Fregoso M. y A. Larqué-Saavedra. 2009. Efecto del ácido Salicílico y dimetilsulfóxido en la floración de Chrysanthemun morifolium (Ramat) Kitamura en Yucatán. Rev. Chapingo Ser. Hortic. 15: 25-31. 